\title{
Selective Branch Prediction Reversal by Correlating with Data Values and Control Flow
}

\author{
Juan L. Aragón', José González', José M. García ${ }^{1}$ and Antonio González² \\ ${ }^{1}$ Dpto. Ingeniería y Tecnología de Computadores \\ Universidad de Murcia \\ 30071 Murcia (Spain) \\ \{jlaragon,joseg,jmgarcia\}@ditec.um.es \\ ${ }^{2}$ Departament d'Arquitectura de Computadors \\ Universitat Politècnica de Catalunya \\ 08034 Barcelona (Spain) \\ antonio@ac.upc.es
}

\begin{abstract}
Branch prediction is one of the main hurdles in the roadmap towards deeper pipelines and higher clock frequencies. This work presents a new approach to enhancing current branch predictors: Selective Branch Prediction Reversal. The rationale behind this proposal is the fact that many branch mispredictions can be avoided if branch prediction is selectively reversed. We present a Branch Prediction Reversal Unit (BPRU) that selectively reverses branch predictions by correlating with the predicted values of the branch inputs, in addition to recent control flow. As a case study, we have included the BPRU in an already proposed branch predictor, the Branch Predictor through Value Prediction (BPVP). The effect is a reduction by half in its original misprediction rate. We have also measured the improvement when the BPRU engine is used in a hybrid scheme composed of a BPVP and a gshare predictors. Results using immediate updates show average reductions in misprediction rate ranging from $7 \%$ to $14 \%$. Performance evaluation of the proposed BPRU in a 20-stage superscalar processor shows an IPC improvement of up to $9 \%$.
\end{abstract}

\section{Introduction}

One of the common ways to increase processor performance relies on reducing the clock cycle. On a given technology, fewer gates per pipeline stage result in higher frequencies. However, this causes an increase in the pipeline depth. For instance, the Intel P6 processor has a pipeline of 10 stages and a first announced clock frequency of $733 \mathrm{MHz}$ at 0.18 microns, whereas the new Intel Pentium 4 was first announced to work at a clock rate of $1.4 \mathrm{GHz}$ with the same technology. To achieve this frequency, the pipeline is lengthened to 20 stages [6].

Deeper pipelines present a serious challenge: the branch misprediction penalty increases since branches take longer to be resolved and thus, the entering to the pipeline of instructions from the correct path is delayed. Even if branch prediction accuracy is quite high, small improvements significantly influence performance, due to the superlinear relationship between prediction accuracy and processor performance [7].

This paper presents a new approach to enhancing current branch predictors: Selective Branch Prediction Reversal. The rationale behind this approach is the fact that many branch mispredictions can be avoided if they are selectively reversed. Inverting some branch predictions was proposed by other authors [14]. However, their approach showed limited performance benefits since the inversion mechanism relied on correlating the inversion with the outcome of recent branches. We propose a Branch Prediction Reversal Unit (BPRU) that reverses branch predictions based on the predicted values of the branch inputs, and the path followed to reach the branch (including the PC of the input producers). Thus, $B P R U$ correlates the inversions with data values and recent control flow.

The $B P R U$ can be combined with any other proposed predictor. As a case study for the application of the $B P R U$, in this work, we use as baseline predictor the Branch Predictor through Value Prediction (BPVP) [8], which is a branch predictor that already correlates predictions with data values. The $B P V P$ was shown to have extremely high prediction accuracy when used in combination with a correlating branch predictor such as the gshare [15], outperforming other contemporary branch predictors. We show that the proposed $B P R U$ can significantly improve the accuracy of the original $B P V P$. On average, the $B P R U$ reduces the misprediction rate of the $B P V P$ by half.

The rest of this paper is organized as follows. Section 2 presents a taxonomy of branch mispredictions. The proposed $B P R U$ is described in Section 3 and Section 4 analyzes its performance. Section 5 presents the related work, and finally, Section 6 summarizes the main conclusions of this work.

\section{Taxonomy of Branch Mispredictions}

This section motivates the inclusion of a Branch 


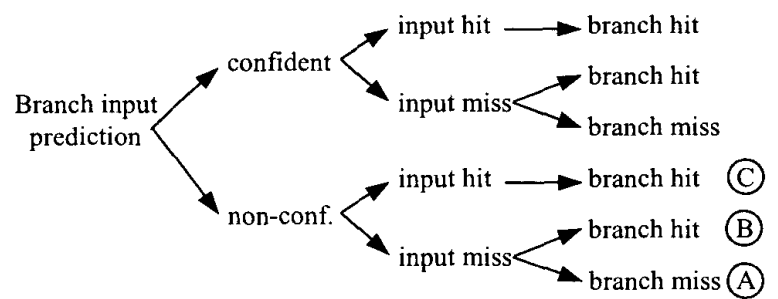

Figure 1. Diagram of the different branch outcomes depending on the input prediction.

\begin{tabular}{|c|c|c|c|c|c|c|}
\hline \multirow{3}{*}{ Benchmark } & \multicolumn{2}{|c|}{ Confident pred. input } & \multicolumn{3}{c|}{ Non-conf. pred. input } \\
\cline { 2 - 7 } & input hit & \multicolumn{2}{|c|}{ input miss } & input hit & \multicolumn{2}{c|}{ input miss } \\
\cline { 2 - 7 } & br. hit & br. hit & br. miss & br. hit & br. hit & br. miss \\
\hline gcc & $42.8 \%$ & $4.4 \%$ & $3.4 \%$ & $11.2 \%$ & $23.8 \%$ & $14.4 \%$ \\
\hline compress & $46.4 \%$ & $0.8 \%$ & $4.6 \%$ & $10.9 \%$ & $22.4 \%$ & $14.9 \%$ \\
\hline go & $27.3 \%$ & $3.9 \%$ & $5.4 \%$ & $16.6 \%$ & $27.4 \%$ & $19.3 \%$ \\
\hline ijpeg & $63.3 \%$ & $1.6 \%$ & $2.5 \%$ & $10.3 \%$ & $13.1 \%$ & $9.1 \%$ \\
\hline li & $45.9 \%$ & $1.6 \%$ & $2.0 \%$ & $5.8 \%$ & $33.9 \%$ & $10.9 \%$ \\
\hline m88ksim & $76.2 \%$ & $0.9 \%$ & $2.6 \%$ & $3.7 \%$ & $11.0 \%$ & $5.5 \%$ \\
\hline perl & $46.8 \%$ & $3.5 \%$ & $3.2 \%$ & $12.9 \%$ & $23.5 \%$ & $10.1 \%$ \\
\hline vortex & $70.6 \%$ & $1.9 \%$ & $1.5 \%$ & $13.4 \%$ & $7.2 \%$ & $5.4 \%$ \\
\hline AVERAGE & $52.4 \%$ & $2.3 \%$ & $3.2 \%$ & $10.6 \%$ & $20.3 \%$ & $11.2 \%$ \\
\hline
\end{tabular}

Table 1. Branch prediction breakdown for an $8 \mathrm{~KB} B P V P$.

Prediction Reversal Unit (BPRU) in a traditional branch predictor. We focus our analysis on the BPVP [8], which predicts branch outcomes by predicting the values of their inputs and performing an early computation of their results according to the predicted values.

Figure 1 establishes a relationship between the behavior of the value predictor and branch predictions. Value predictions can be split into confident and non-confident, depending on the confidence counter of the entry being used $^{1}$. Each of them can result in a branch input hit or a branch input miss. A value prediction hit causes a branch prediction hit. However, a value prediction miss does not necessarily cause a branch miss. For instance, if a branch checks whether the input value is different from zero, any predicted input value but zero will cause a branch hit.

Table 1 quantifies the frequency of the different cases described in Figure 1 for the whole SpecInt95 benchmark suite. The $B P V P$ uses an $8 \mathrm{~KB}$ stride predictor as value predictor. Section 4 further details the experimentation process. First of all, the value predictor provides $57.9 \%$ of confident predictions and $42.1 \%$ of non-confident ones. Most of the confident input predictions are correct $(52.4 \%$ over $57.9 \%$ ), and just a minor percentage cause branch misses $(3.2 \%$ over $57.9 \%)$. Furthermore, for the nonconfident input predictions, $31.5 \%$ over $42.1 \%$, lead to value mispredictions. We also see that the majority of the total branch mispredictions come from these non-

\footnotetext{
${ }^{1}$ Value predictor entries have a confidence field, usually implemented as a saturating counter, in order to assign confidence to predictions [12].
}

confident input mispredictions $(11.2 \%$ over $14.4 \%)$. All benchmarks follow this trend, which suggests a correlation between branch mispredictions and value predictions: most branch misses come from non-confident predicted inputs and only a few branch mispredictions come from confident ones. However, in order to reverse branch predictions, not only the confidence counters of the value predictor should be taken into account. If all branch predictions based on non-confident input predictions were reversed, the overall accuracy would be degraded.

\section{Branch Prediction Reversal Mechanism}

This section analyzes alternative parameters that may be used in a branch reversal mechanism and then, the proposed implementation of the $B P R U$ is described.

\subsection{Quantitative Analysis of the Branch Reversal Mechanism}

We have performed an off-line analysis in order to gain some insight into the processor parameters that provide a better correlation with branch mispredictions. The following parameters have been independently examined:

a) The predicted value of the branch input.

b) The PC of the branch input producer.

c) The predicted branch input and the branch PC.

d) The predicted branch input and the PC of the branch input producer.

e) The predicted branch input, the PC of the branch input producer and the path followed to reach the branch.

We have run the entire SpecInt95 suite using a modified version of the sim-safe simulator [2]. Then, the occurrences of cases $A, B$ and $C$ (see Figure 1) are measured for the five scenarios, assuming unbounded storage resources. For those parameter values for which Equation (1) is fulfilled, the branch prediction is reversed.

occurrences in $A>$ (occurrences in $B+$ occurrences in $C$ ) (1)

Thus, a new misprediction rate is obtained, which shows the potential of reversing the branch prediction considering this a priori information. More details about these experiments can be found in [1]. Figure 2 shows the new misprediction rate for $g c c$, go, ijpeg and $l i$

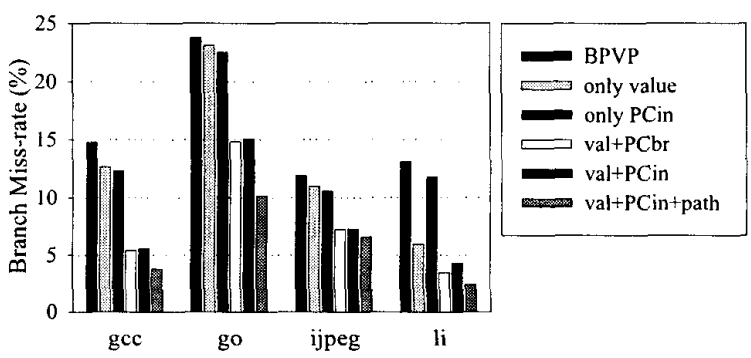

Figure 2. Potential misprediction rate using branch inversion 


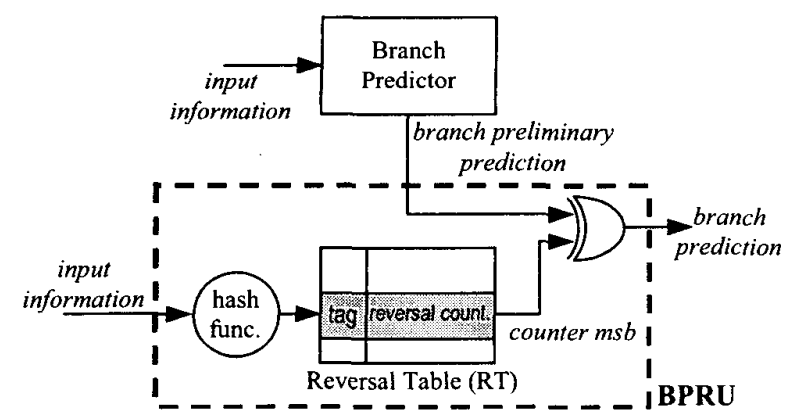

Figure 3. Block diagram of the $B P R U$.

applications for the five evaluated scenarios. The underlying branch predictor is the BPVP using a stride value predictor with an unrealistic size of $1 \mathrm{MB}$ in order to isolate the potential of our proposal from the performance of the value predictor. It can be observed that the approach (e) is the best one. It reduces the $B P V P$ misprediction rate by half for all benchmarks. These results show the potential of branch prediction reversal to enhance the performance of branch predictors when data values and control flow information are taken into account.

\subsection{Branch Prediction Reversal Unit (BPRU)}

This section presents the implementation of the Branch Prediction Reversal Unit (BPRU). As a case study, we show how it works in conjunction with the BPVP predictor, although this unit could be included in any branch predictor.

Figure 3 depicts the block diagram of the $B P R U$. It consists of a Reversal Table (RT) and the logic necessary for making the reversal of the preliminary branch outcome. Each entry of the $R T$ stores a reversal counter, which is an up/down saturating counter, and a tag. The $R T$ is accessed when the branch is predicted, by hashing some processor state information. The most significant bit of the counter of the corresponding $R T$ entry indicates whether the branch outcome is reversed. Once the correct branch outcome is computed, the $R T$ entry is updated, incrementing the counter if the preliminary branch outcome was incorrect, and decreasing the counter otherwise.

Figure 4 depicts the block diagram of the $B P R U$ when it is integrated along with the $B P V P$ predictor. Details about how the $B P V P$ works can be found in [8]. We refer to this new scheme as $B P V P+B P R U$. According to the analysis of the previous section, the most effective approach to reversing branch predictions is to correlate with the predicted value, the PC of the branch input producer and the path followed to reach the branch. The first and the second parameters along with a nonconfidence signal are forwarded from the $B P V P$ to the $B P R U$. In addition, the BPRU maintains a Path History

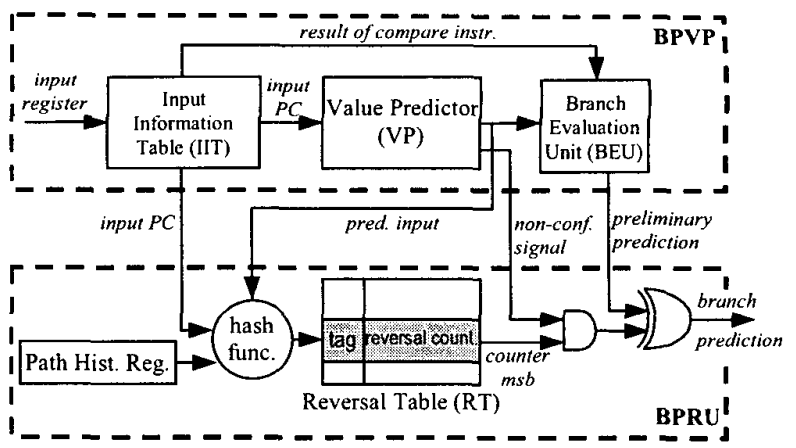

Figure 4. Block diagram of the $B P R U$ integrated along with the $B P V P$.

Register $(P H R)$, which stores the path followed to reach the branch. For each fetched control-flow instruction (conditional or unconditional), the $P H R$ is shifted 2 bits to the left and the 2 least significant bits of the PC are shifted in. The $R T$ is indexed by hashing the PC of the branch input producer, the predicted value and the $P H R$. Nevertheless, for other branch predictors, different information could be used, such as the values of some particular registers, the branch $\mathrm{PC}$, history of recent outcomes, etc.

Conflicts in the $R T$ are one of the major problems that may limit the $B P R U$ performance [1]. We observed that the use of tags alleviates destructive aliasing, obtaining higher performance than a non-tagged $R T$ of the same size, despite of the space occupied by the tags. Besides, the replacement policy of the $R T$ has to be carefully selected. Our replacement policy gives priority to entries with lower values in their reversal counter.

\section{Experimental Results}

This section analyzes the performance of the proposed $B P R U$ engine when it is integrated along with the $B P V P$. We also present results for a hybrid mechanism composed of two correlating predictors: bimodal (2bit) [19] and gshare [15]. Thus, the evaluated hybrid predictors are: $B P V P+B P R U+$ gshare, $B P V P+$ gshare, and $2 b i t+$ gshare ${ }^{2}$.

\subsection{Simulation Methodology}

We have considered the five programs from the SpecInt95 benchmark suite that exhibit the highest misprediction rates. Table 2 shows for each benchmark the input set, the number of dynamic instructions and the number of conditional branches. All benchmarks were compiled with maximum optimizations (-O4 -migrate) by the Compaq Alpha compiler, and they were run until

\footnotetext{
${ }^{2}$ The first and the second predictors use the selector proposed in [8], whereas the 2bit+gshare uses the selector proposed in [15]. For each case, we chose the selector that produced the best results.
} 
completion using the SimpleScalar/Alpha v3.0 tool set [2].

\begin{tabular}{|l|c|c|c|}
\hline Benchmark & Input Set & $\begin{array}{c}\text { \# dyn. Instr. } \\
\text { (in Mill.) }\end{array}$ & $\begin{array}{c}\text { \# dyn.cond. } \\
\text { branch (Mill) }\end{array}$ \\
\hline compress & 40000 e 2231 & 169.6 & 12.6 \\
\hline gcc & genrecog.i & 145.4 & 19.3 \\
\hline go & 99 & 145.6 & 15.4 \\
\hline ijpeg & specmun -qual 45 & 166.0 & 9.4 \\
\hline li & 7 queens & 242.7 & 32.0 \\
\hline
\end{tabular}

Table 2. Benchmark characteristics

\subsection{Results for Immediate Updates}

The first set of experiments update prediction tables immediately, in order to evaluate the potential of the selective reversal mechanism when it is isolated from other aspects of the microarchitecture (using the sim-safe simulator). We first measure the misprediction rate of the $B P V P+B P R U$ predictor for different sizes. For each configuration, half of the total size is devoted to the $B P V P$ and the other half to the $B P R U$. The $R T$ is implemented as an 8-way associative table using 13 bits for tags and 3 bits for the reversal counters. All the experiments compare predictors of the same total size, including the space occupied by tags and counters.

Figure 5 shows the results. It can be observed that $B P V P+B P R U$ significantly outperforms $B P V P$ for all benchmarks and all evaluated sizes. On average, the $B P R U$ reduces the misprediction rate of the $B P V P$ by half for $32 \mathrm{~KB}$ capacity. Besides, as the total predictor size grows, the difference between both misprediction rates becomes higher, which shows that the $B P R U$ exploits other type of correlations not included in the BPVP.

The misprediction rate of the $B P V P$ is not impressive, since this predictor was designed to be used in conjunction with a correlating branch predictor [8]. Figure 6 shows the misprediction rates for the hybrid $B P V P+B P R U+$ gshare, $B P V P+$ gshare and $2 b i t+$ gshare predictors. More details about the configurations used can be found in [1].

First, the $B P V P+B P R U+$ gshare outperforms the $B P V P+$ gshare for all benchmarks and for all size configurations excepting compress, for which both show about the same performance. The $B P V P+B P R U+$ gshare with a size of $36 \mathrm{~KB}$ obtains, on average, a similar misprediction rate than the $B P V P+$ gshare of $128 \mathrm{~KB}$. Second, the combination of $B P V P+B P R U+$ gshare significantly outperforms the $2 b i t+g s h a r e$ for all size configurations. On average, the $B P V P+B P R U+$ gshare with a total size of $9 \mathrm{~KB}$ has about the same misprediction rate $(7.7 \%)$ as the 2 bit + gshare of $128 \mathrm{~KB}(7.5 \%)$. Summarizing, on average the $B P V P+B P R U+$ gshare reduces the misprediction rate by a factor that ranges from $7 \%$ to $14 \%$ with respect to the $B P V P+$ gshare, and from $24 \%$ to $35 \%$ with respect to the 2 bit + gshare.

Finally, we note that the potential of the $B P R U$ is
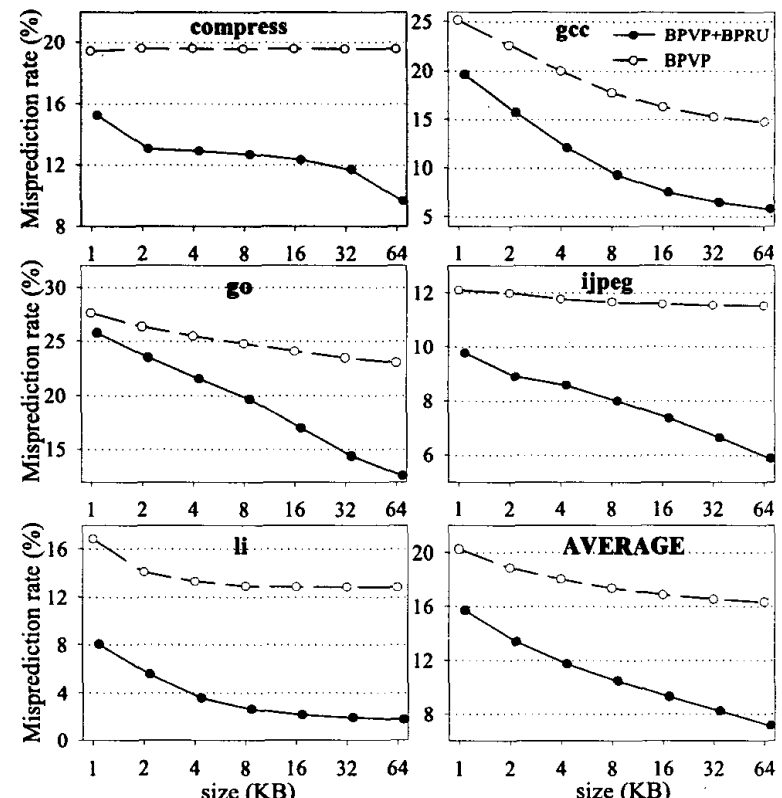

Figure 5. Branch misprediction rates for $B P V P+B P R U$ and $B P V P$ predictors for five SpecInt95 applications as well as the arithmetic mean.
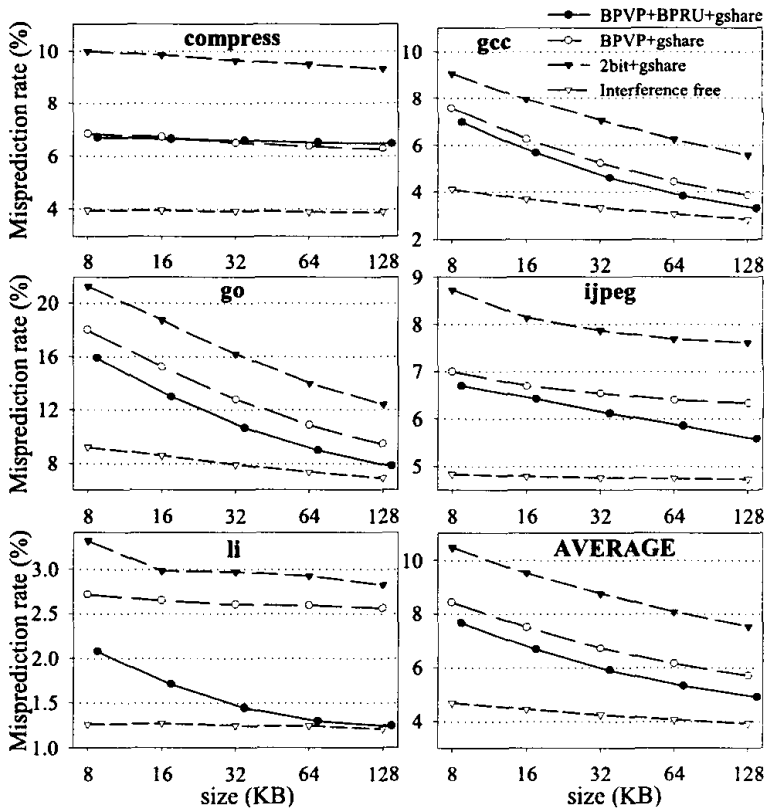

Figure 6. Branch misprediction rates for $B P V P+B P R U+$ gshare, $B P V P+$ gshare, 2bit + gshare and $B P V P+B P R U+$ gshare with an interference-free $R T$.

limited by destructive aliasing when accessing the $R T$. This can be observed by looking at the misprediction rate of the $B P V P+B P R U+$ gshare using an interference-free $R T$. The unbounded $R T$ provides huge improvements for 
all benchmarks. For instance, in the go program, the miss rate of an $8 \mathrm{~KB} \mathrm{BPVP+gshare} \mathrm{drops} \mathrm{from} 18 \%$ to $9 \%$ when a $B P R U$ with an interference-free $R T$ is included. This shows the potential of the proposed branch reversal mechanism as well as an opportunity for improvement by using better indexing schemes to access the $R T$.

\subsection{Results for Realistic Updates}

This section presents an evaluation of the proposed $B P R U$ in a dynamically-scheduled superscalar processor. Details of the simulated superscalar pipeline are described in Table 3. In addition, the original sim-outorder simulator pipeline has been lengthened to 20 stages, following the pipeline scheme of the Pentium 4 processor [6].

\begin{tabular}{|c|l|}
\hline Fetch engine & $\begin{array}{l}\text { Up to } 8 \text { instructions/cycle, 2 taken branches, } \\
8 \text { cycles misprediction pcnalty. }\end{array}$ \\
\hline Execution engine & $\begin{array}{l}\text { Issues up to } 8 \text { instructions/cycle, 128-entries } \\
\text { reorder buffer, 64-entries load/store queue. }\end{array}$ \\
\hline Functional Units & $\begin{array}{l}8 \text { integer alu, 2 integer mult, 2 memports, } \\
8 \mathrm{FP} \text { alu, 1 FP mult. }\end{array}$ \\
\hline L1 Instr-cache & $\begin{array}{l}128 \mathrm{~KB}, 2 \text {-way set associative, 32 bytes/line, } \\
1 \text { cycle hit latency. }\end{array}$ \\
\hline L1 Data-cache & $\begin{array}{l}128 \mathrm{~KB}, 2 \text {-way set associative, 32 bytes/line, } \\
1 \text { cycle hit latency. }\end{array}$ \\
\hline L2 unified cache & $\begin{array}{l}512 \mathrm{~KB}, 4 \text {-way set associative, 32 bytes/line, } \\
6 \text { cycles hit latency, 18 cycles miss latency. }\end{array}$ \\
\hline M cmory & $\begin{array}{l}8 \text { bytes/line, virtual memory } 4 \mathrm{~KB} \text { pages, } \\
30 \text { cycles TLB miss. }\end{array}$ \\
\hline
\end{tabular}

Table 3. Simulated superscalar pipeline parameters.

Figure 7 shows the IPC obtained for each benchmark when using the $B P V P+B P R U+$ gshare, $B P V P+$ gshare and $2 b i t+g s h a r e$ predictors for three different sizes. The latency considered for the $2 b i t+g s h a r e$ is one cycle, that is, the branch prediction is made during the fetch stage. The latency considered for the $B P V P+B P R U$ is 3 cycles, since the $B P V P$ has to perform several table accesses to provide the prediction ${ }^{3}$ [8]. We can observe that the addition of the $B P R U$ results in a significant speedup for all cases. The average IPC obtained with the

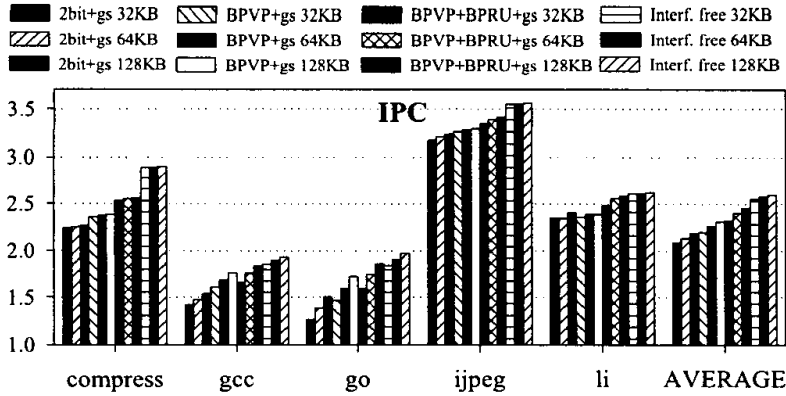

Figure 7. IPC for $B P V P+B P R U+$ gshare, $B P V P+$ gshare and 2 bit + gshare for different predictor sizes.

${ }^{3}$ To reach this latency, accesses to the different tables can be pipelined by adding latches in between.
$B P V P+B P R U+g s h a r e$ predictor is significantly higher than the IPC of the 2 bit + gshare (average speedups of $13 \%, 14 \%$ and $14 \%$ for $32 \mathrm{~KB}, 64 \mathrm{~KB}$ and $128 \mathrm{~KB}$ respectively). Also, a $B P V P+B P R U+$ gshare of about 32 $\mathrm{KB}$ achieves the same performance as a $B P V P+$ gshare of $128 \mathrm{~KB}$.

Table 4 shows the speedup obtained by $B P V P+B P R U+$ gshare with respect to $B P V P+$ gshare and $2 b i t+g$ share for a total predictor size of $64 \mathrm{~KB}$.

\begin{tabular}{|c|c|c|c|c|c|c|c|}
\hline Baseline & BPRU & compress & gcc & go & ijpeg & li & AVG. \\
\hline \multirow{2}{*}{$\begin{array}{c}\text { BPVP+ } \\
\text { gshare }\end{array}$} & realistic & 1.07 & 1.04 & 1.09 & 1.03 & 1.07 & 1.06 \\
\cline { 2 - 8 } & Interf.free RT & 1.22 & 1.11 & 1.18 & 1.08 & 1.11 & 1.14 \\
\hline $\begin{array}{c}2 \text { bit+ } \\
\text { gshare }\end{array}$ & realistic & 1.19 & 1.13 & 1.25 & 1.05 & 1.09 & 1.14 \\
\cline { 2 - 8 } & Interf.free RT & 1.29 & 1.28 & 1.38 & 1.10 & 1.12 & 1.23 \\
\hline
\end{tabular}

Table 4. Speedup for a total size of $64 \mathrm{~KB}$.

The average speedup of the $B P V P+B P R U+$ gshare over $B P V P+g s h a r e$ is $6 \%$. Go is the benchmark that obtains the highest speedup (9\%). Comparing $B P V P+B P R U+$ gshare with 2 bit + gshare, the average speedup is about $14 \%$. The benchmark that obtains the best speedup is again go $(25 \%)$. Finally, the speedup of the $B P R U$ with an interfererence-free $R T$ is very high, specially for compress, $g c c$ and $g o$. For a size of $64 \mathrm{~KB}$, the average speedups over $B P V P+$ gshare are $22 \%, 11 \%$ and $18 \%$ respectively.

\section{Related Work}

The vast majority of branch predictors rely on the fact that the outcome of a branch may correlate with its own history [19][20], the behavior of previous branches [15][19], or the path followed by the program [16]. Some other works have focused on improving the performance of those predictors by avoiding aliasing [4][18] or by combining different branch predictors [5][15].

On the other hand, several studies have shown that some instructions generate data values that follow predictable patterns [13][17]. Therefore, value prediction has been mainly applied to data value speculation [3][12]. The aim of these proposals is to overcome the serialization imposed by data dependences.

In [17], the potential of improving branch prediction accuracy by using data value prediction is suggested but no particular mechanism is proposed. In [8], it is proposed the $B P V P$ predictor, which correlates branch predictions with data values, obtaining a very high accuracy when it is used along with a correlating branch predictor. In [10], it is proposed a branch predictor which correlates with data values to index a prediction table. The scheme also includes a Rare Event Predictor, for the exceptional cases.

In [11], a branch confidence estimator is proposed, and although it is suggested that can be used for branch reversal, neither a particular implementation nor a miss rate evaluation is presented. In [9], different branch 
confidence estimators are proposed and, in [14], they are evaluated when used for Selective Branch Inversion. All proposed confidence estimators are based on correlating with recent branch outcomes and the branch PC, without correlating with other processor parameters such as data values. The results showed average misprediction reductions by a factor of $5 \%-7 \%$ over a $2 b i t+$ gshare (named mcfarling in that work), which is lower than the reduction we present in this work $(7 \%-14 \%$ achieved by the $B P R U+B P V P+$ gshare over $B P V P+$ gshare, which, in turn, is a better predictor than the 2 bit + gshare).

\section{Conclusions}

In this paper we have proposed a Selective Branch Prediction Reversal mechanism as an effective approach to improving branch prediction accuracy. It relies on the fact that many branch mispredictions can be avoided if they are selectively reversed based on some processor parameters. We have evaluated several parameters and showed that the result of a branch prediction can be correlated with the predicted data value of the branch input, path history and the PC of the branch input producer. We have proposed a Branch Prediction Reversal Unit $(B P R U)$ that selectively reverses particular branches likely to be mispredicted, based on the above parameters.

As an example of its functionality, we have integrated the $B P R U$ with the $B P V P$ predictor, which on average results in a reduction in misprediction rate by half. In addition, we have compared the hybrid $B P V P+B P R U+$ gshare against both the $B P V P+$ gshare and the 2 bit + gshare predictors. Results using immediate updates show average reductions of misprediction rates by a factor that ranges from $24 \%$ to $35 \%$ over $2 b i t+$ gshare, and from $7 \%$ to $14 \%$ over $B P V P+$ gshare.

We have also evaluated the proposed $B P V P+B P R U+$ gshare predictor for a superscalar processor with a 20 stage pipeline using realistic table updates and prediction latencies. Results show average speedups of $6 \%$ (up to $9 \%$ for some applications) over BPVP+gshare and 14\% (up to $25 \%$ ) over $2 b i t+g$ share. Results have also shown that the potential performance of the $B P R U$ is limited by destructive aliasing. This suggests an opportunity for improvement by exploring other indexing schemes to access the Reversal Table.

\section{Acknowledgements}

This work has been supported by the project TIC20001151-C07-03 of the Plan Nacional of $I+D+I$ of the Spanish Ministry of Science and Tech., by the Spanish CICYT under grant TIC98-0511 and Fundación Séneca.

\section{References}

[1] J.L. Aragón, J. González, J.M. García and A. González. "Selective Branch Prediction Reversal by Correlating with
Data Values and Control Flow". Tech.Report \#UM-DITEC2000-08, University of Murcia, 2000. http://ditec.um.es/ reportsFiles/ditec/publics/UM-DITEC-2000-8.pdf

[2] D. Burger and T.M. Austin. "The SimpleScalar Tool Set, Version 2.0". Technical Report \#1342, University of Wisconsing-Madison, Computer Sciences Department, 1997.

[3] B. Calder, G. Reinman and D.M. Tullsen. "Selective Value Prediction". Proc. of the Int. Symp. on Comp. Arch., 1999.

[4] P.Y. Chang, M. Evers and Y.N. Patt. "Improving Branch Prediction Accuracy by Reducing Pattern History Table Interference". Proc. of the Int. Conf. on P.A.C.T., 1996.

[5] M. Evers, P.Y. Chang and Y.N. Patt. "Using Hybrid Branch Predictors to Improve Branch Prediction Accuracy in the Presence of Context Switches". Proc. of the I.S.C.A., 1996.

[6] P.N. Glaskowsky. "Pentium 4 (Partially) Previewed". Microprocessor Report (Microdesign Res.), August 2000.

[7] J. González and A. González. "The Potential of Data Value Speculation to Boost ILP". Proc. of the Int. Conf. on Supercomputing, pp. 21-28, 1998

[8] J. González and A. González. "Control-Flow Speculation through Value Prediction for Superscalar Processors". Proc. of the Int. Conf. on Parallel Arch. and Compil. Tech., 1999.

[9] D. Grunwald, A. Klauser, S. Manne and A. Pleszkun. "Confidence Estimation for Speculation Control". Proc. of the Int. Symp. on Computer Architecture, 1998.

[10] T.H. Heil, Z. Smith and J.E. Smith. "Improving Branch Predictors by Correlating on Data Values". Proc. of the Int. Symp. on Microarchitecture, 1999.

[11] E. Jacobsen, E. Rotenberg and J.E. Smith. "Assigning Confidence to Conditional Branch Predictions". Proc. of the Int. Symp. on Microarchitecture, 1996.

[12] M.H. Lipasti and J.P. Shen. "Exceeding the Dataflow Limit via Value Prediction". Proc. of the Int. Symp. on Microarchitecture, pp. 226-237, 1996.

[13] M.H. Lipasti, C.B. Wilkerson and J.P. Shen. "Value Locality and Load Value Prediction". Proc. of the Int. Conf. on Architect. Support for Prog. Lang. and Oper. Systems, 1996.

[14] S. Manne, A. Klauser and D. Grunwald. "Branch Prediction using Selective Branch Inversion". Proc. of the Int. Conf. on Parallel Architectures and Compilation Tech., 1999.

[15] S. McFarling. "Combining Branch Predictors". Tech. Report \#TN-36. Digital Western Research laboratory, 1993.

[16] R. Nair. "Dynamic Path-Based Branch Correlation". Proc. of the Int. Symp. on Microarchitecture, pp. 15-23, 1995.

[17] Y. Sazeides and J.E. Smith. "Modeling Program Predictability". Proc. of the Int. Symp. on Computer Architecture, pp. 73-84, 1998.

[18] E. Sprangle, R.S. Chappell, M. Alsup and Y.N. Patt. "The Agree Predictor: A Mechanism for Reducing Negative Branch History Interference". Proc. of the I.S.C.A., 1997.

[19] J.E. Smith. "A Study of Branch Prediction Strategies". Proc. of the Int. Symp. on Computer Architecture, 1981.

[20]T.Y. Yeh and Y.N. Patt. "A Comparison of Dynamic Branch Predictors that Use Two Levels of Branch History". Proc. of the Int. Symp. on Comp. Architecture, pp. 257-266, 1993. 